\title{
Computational Insights into the Role of the Frontiers Orbital in the Chemistry of Tridentate Ligands
}

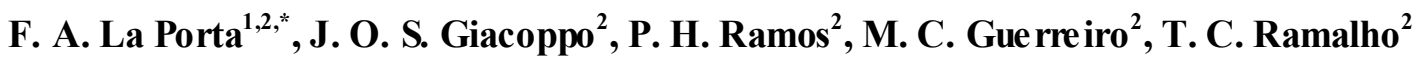 \\ ${ }^{1}$ Department Physical Chemistry, Paulista State University, CEP 14801-970, Araraquara, SP, Brazil \\ ${ }^{2}$ Department of Chemistry, Federal University of Lavras, CEP 37200-000, Lavras-M G, Brazil
}

\begin{abstract}
The FERMO concept emerges as a powerful and innovative implement to investigate the role of molecular orbitals applied in the description of breakage and formation of chemical bonds. In this work, Hartree-Fock (HF) and Density Functional Theory (DFT) calculations were carried out for a series of four tridentate ligands and their behavior was analyzed using molecular orbital (MO) energies. It was observed that HOMO energies are inadequate to describe the Pearson's Hard and Solf acid-base (HSAB) pricinple behavior of these compounds. By using the frontier effective-for-reaction molecular orbital (FERMO) concept, the reactions that are driven by HOMO, and those that are not, can be better explained, independent of the calculation method used, since both HF and Kohn-Sham methodologies lead to the same FERMO.
\end{abstract}

Keywo rds FERMO, TridentateLigands, HSAB, HOMO-LUMO, Molecular Orb ital

\section{Introduction}

Among the various organic ligands that are used in the synthesis ofcoordination compounds, several studies have been developed with tridentate ligands[1-6].These ligands coordinate to the metal through three atoms and spectroscopic properties are of interest in various areas ofscience such as molecular magnetic materials, lasers in optical fiber and luminescent sensors[7-12]. Molecular orbitals (MOs) and their properties, like energies and symmetries, are very useful for chemists. According to Fukui, the MO properties have used the frontier electron density for predicting the most reactive position in $\pi$-electron systems[13-15]. Hoffmann and Woodward set out orbital symmetry rules to explain several types of reactions in conjugated systems, and the frontier MOs gained importance for the better understanding of chemical reactions. The concept of frontier orbital, introduced by Fukui around 1952, relates reactivity with the properties of two molecular orbitals: HOMO and LUMO. One application of the HOMO-LUMO argument is the description of the acid-base behavior of compounds [13-17].

Thus, given the limitations of HOMO-LUMO argument and the new approaches proposed in the literature to understand the chemical reactivity, one must move forward the role of molecular orbitals in chemistry, giving rise to a new idea: the Frontier Effective-for-Reaction Molecular

* Corresponding author:

felipe_laporta@yahoo.com.br(F. A. La Porta)

Published online at http://journal.sapub.org/chemistry

Copyright (C) 2012 Scientific \& Academic Publishing. All Rights Reserved
Orbital (FERMO)[18-28].

The FERMO concept comes from a dose of intuition, together with the criteria for the composition and location to correctly determine the reactant molecular orbital. This concept can be understood as a complement to the HOMO-LUMO argument. Another important feature in FERMO is that both HF and Kohn-Sham orbitals take the same conclusions about reactivity[23-30].

In line with those expectations, the goal of the current work is devoted to investigating which MO is the best for describing the reactivity for some systems formed by tridentate ligands, such as the 2-6 pyridinedicarbo xy lic acid in light of the FERMO concept.

\section{Computional Methods}

In recent years, theoretical methods based on the Density Functional Theory (DFT) have emerged as an alternative to traditional $a b$ initio methods in the study of structure and reactivity of chemical systems[31,32]. In this work, all calculations were carried out with the Gaussian03 package[33]. Each tridentate ligands of all 5 compounds was fully optimized using DFT with the B3LYP functional $[34,35]$ employing the $6-31 \mathrm{G}(\mathrm{d}, \mathrm{p})$ basis set. No symmetry constraint was imposed during the optimization process. Those optimized geometries were used in all subsequent calculations. This theoretical level was also used for the frequency calculations. Furthermo re, ab initio MP2 energy calculations were computed using the 6-31G(d,p) bas is set in gas-phase. The MO figures were prepared using the Gauss View 2.1 package[33] with a contour value of 0.020 .

For the calculations, the iron-dipicolinic acid complexes were calculated and characterized by DFT. The energy 
minima were identified by building potential energy surfaces, obtained along the reaction pathway at the B3LYP/ $6-31 \mathrm{~g}(\mathrm{~d}, \mathrm{p})$ and (8s6p4d1f) level[34]. Furthermore, after each optimization, the nature of the stationary point was established by calculating and diagonalizing the Hessian matrix (force constant matrix). The unique imag inary frequency associated with the transition vector (TV) was characterized. This computational procedure has been used previously in similar systems with success[36-39]. For all calculations of the structures of this work were calculated in gas phase and was not considered the effect of the solvent.

\section{Results and Discussion}

In this work, we report the study to show how the FERMO concept can be used to explain the hard and soft behavior for the tridentate ligands (Figure 1). These ligands were chosen because a large number of their experimental and theoretical studies are available in the literature [40-47]. Therefore, these investigations lead to new perspectives and ideas about the reactivity.

Reactions involving electron donation and acceptance are related to MO energies, since electrons are occupying and will occupy a MO and a frontier one, as stated by Fukui. By exploration of Pearson's hard-soft acid-base (HSAB) concept, Klop man proposed the concept of charge or frontier controlled reactions[48,49]. Following the Pearson's principle that soft molecules have smaller HOMO-LUMO gaps when compared with hard ones, it can be stated that soft reaction sites in a molecule will have a smaller FERMO-LUMO gap than harder ones. Clearly, the HOMO-LUMO gap itself can not describe the hardness difference between the two binding sites in the same molecule. The very chemically intuitive Frontier Effective-for-Reaction Molecular Orbital (FERMO) concept was introduced to solve HOMO-LUMO limitations and better exp lain the chemical behavior of mo lecules.<smiles>O=C(O)c1cccc(C(=O)O)n1</smiles>

(1)<smiles>NCCNCCN</smiles>

(3)<smiles>O=C(O)c1cc(O)cc(C(=O)O)n1</smiles>

(2)<smiles>NCc1ccc2cccc(O)c2n1</smiles>

(4)
Figure 1. Structure of the tridentate ligands

Table 1 shows the FERMO-LUMO energy gaps for the studied compounds. Thus, this concept can be understood as a comp lement to the HOMO-LUMO argu ment.

In the FERMO concept, $\mathrm{MO}$ composition and shape are taken into account to identify the MO that will actually be involved in a given reaction. A molecule could have as many FERMOs as it has reactions sites and it could be the HOMO or any other frontier MO. Reactions involving electron donation and acceptance are related to MO energies, since electrons are occupying and will occupy a $\mathrm{MO}$ and a frontier one, as stated by Fukui[13-15]. Based on the MO location and composition, we can identify the FERMOs for 5 tridentate ligands studied, shown in Figure 2. The MO shape and the atomic composition are very important parameters for analy zing FERMO. Upon analyzing orbital localization and composition more deeply, it can be observed that there is a MO with energy quite close to the HOMO energy value and with large ligands contribution (Table 1). Thus, it is supposed that this orbital could better describe the acid/base behavior than HOMO.

Table 1. 1/2 FERMO-LUMO gap for studied molecules (all values in eV), Tridentate ligands contribution (\%), MO numeration and orbital energy for all molecules studied using MP2/6-31 $\mathrm{g}(\mathrm{d}, \mathrm{p})$ in gas-phase

\begin{tabular}{|c|c|c|c|c|c|}
\hline & Molecules & Contribution (\%) & $1 / 2$ FERMO-LUMO gap & MO numeration & Energy (a.u.) \\
\hline \multirow{4}{*}{1} & HOMO & - & - & 43 & $-0,375$ \\
\hline & N-FERMO ${ }^{\mathrm{a}}$ & 54,80 & 6,83 & 41 & $-0,434$ \\
\hline & $\mathrm{COO}^{-} \mathrm{FERMO}^{\mathrm{b}}$ & 65,64 & 7,16 & 40 & $-0,457$ \\
\hline & $\mathrm{COO}^{-} \mathrm{FERMO}^{\mathrm{b}}$ & 84,24 & 7,43 & 39 & $-0,478$ \\
\hline \multirow{4}{*}{2} & HOMO & - & - & 47 & $-0,376$ \\
\hline & N-FERMO ${ }^{\mathrm{a}}$ & 56,58 & 6,83 & 45 & $-0,437$ \\
\hline & $\mathrm{COO}^{-} \mathrm{FERMO}^{\mathrm{b}}$ & 80,00 & 7,13 & 44 & $-0,458$ \\
\hline & $\mathrm{COO}^{-} \mathrm{FERMO}^{\mathrm{b}}$ & 22,67 & 7,43 & 43 & $-0,480$ \\
\hline \multirow{4}{*}{3} & HOMO & - & - & 29 & $-0,350$ \\
\hline & N-FERMO ${ }^{\mathrm{a}}$ & 68,76 & 7,89 & 29 & $-0,350$ \\
\hline & N-FERMO ${ }^{b}$ & 69,01 & 8,19 & 28 & $-0,371$ \\
\hline & N-FERMO ${ }^{b}$ & 82,66 & 8,25 & 27 & $-0,375$ \\
\hline \multirow{4}{*}{4} & HOMO & - & - & 46 & $-0,280$ \\
\hline & O-FERMO ${ }^{\mathrm{a}}$ & 26,87 & 5,12 & 46 & $-0,280$ \\
\hline & N-FERMO ${ }^{\mathrm{a}}$ & 31,87 & 5,58 & 45 & $-0,314$ \\
\hline & N-FERMO ${ }^{b}$ & 60,70 & 6,56 & 43 & $-0,387$ \\
\hline
\end{tabular}

*(a) So ff site; (b) Hard site 


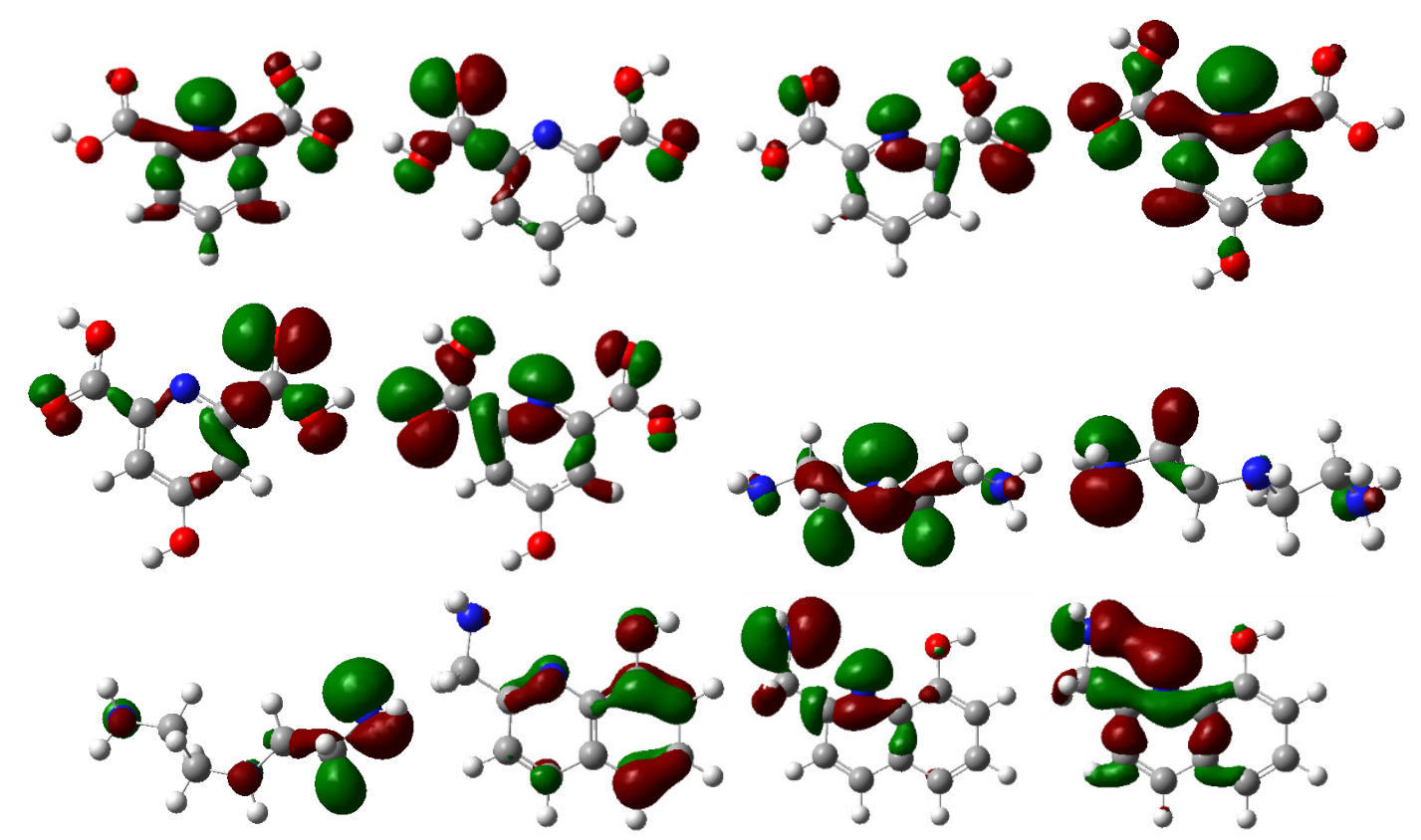

Figure 2. Surface plots for FERMOs at the MP2 level for the tridentate ligands

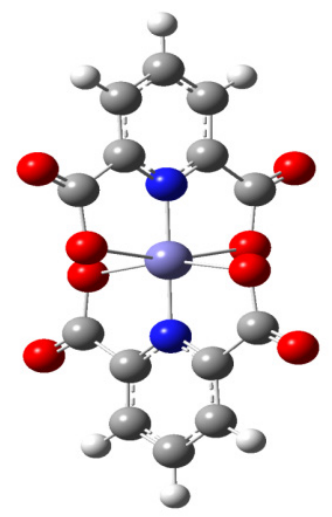

Figure 3. Model structure Fe-PDC

Another important point is the correspondence between FERMO shapes and the geometry of these ligands in coordination compounds, independent of the calculation method used, since both HF and Kohn-Sham methodologies lead to the same FERMO[24]. The complexation of metallic ions with the 2-6 pyridinedicarboxylic acid (Figure 3 ) has been extensively studied[42,44-37]. This is due to the fact that PDC is a versatile chelating agent that can act as a bidentate, tridentate, meridian or bridge bonding with different metallic ions[44-47]. PDC also presents other important characteristics, such as low toxicity and its diversified biological activity being present in many natural products such as oxidative degradation product of vitamins, coenzymes and alkaloids[50,51]. The use of organic iron complexes in catalysis has been pointed to as advantageous by some authors, considering the stabilization of the iron in a wider $\mathrm{pH}$ range in relation to that in the absence of complexes. Another advantage is that most of the chelating agents are natural products that are easily degraded by microorganis ms. Therefore, this alteration in the Fenton reaction does not represent an environmental concern and can be directly compatible with the biodegradation process $[52,53]$. Thus, in order to investigate this possibility, we have performed thermodynamic stability calcu lations for this scenario.

Most $\mathrm{Fe}^{3+}$ complexes with tridentate ligands are also octahedral, and the complex with $\mathrm{d}^{9}$ configuration can generate the Jahn-Teller distortion, i.e., distortion of the octahedral complex. That observation is in very good agreement with experimental studies [54].

The FTIR (Figure 4) showed bands that confirm the formation of the complex compared with the free ligand spectrum. In the IR spectra, the complex is observed at 1686 $\mathrm{cm}^{-1}$, a vibration regarding the carboxylate ion $\left(\mathrm{COO}^{-}\right)$. The dis appearance of the deformations corresponding to $\mathrm{C}-\mathrm{OH}$ in and out of the plane at $1418 \mathrm{~cm}^{-1}$ and $923 \mathrm{~cm}^{-1}$ therefore this indicates the deprotonation of the carboxylic group in the formation of the complex. The strong absorption in the region of $3100-2500 \mathrm{~cm}^{-1}$ in the non-coordinated ligand is due to the $\mathrm{COOH}$ group stretching, superimposed with the $\mathrm{C}-\mathrm{H}$ band. The theoretical results are in good agreement with the experimental data for complex iron-PDC $[46,55]$.

Non-bonded interactions play an important role in the complex stabilization[56]. For instance, studies with 69 additional published crystal structures of organic, inorganic, and organometallic compounds containing divalent sulfur ( $\mathrm{S}$ bonded to two ligands, $\mathrm{Y}$ and $\mathrm{Z}$, different from $\mathrm{H}$ ) revealed that, in the lattice, electrophiles tend to approach the sulfur atom roughly $20^{\circ}$ from a line perpendicular to the Y-S-Z ato ms plane, whereas nucleophiles tend to approach approximately along the extension of one of the sulfur covalent bonds[57,58]. We believe that these regularities portray features of the electron distribution and indicate the preferred direction of approximation of electrophiles and nucleophiles, as well as the preferred direction for non-bonded interactions. 


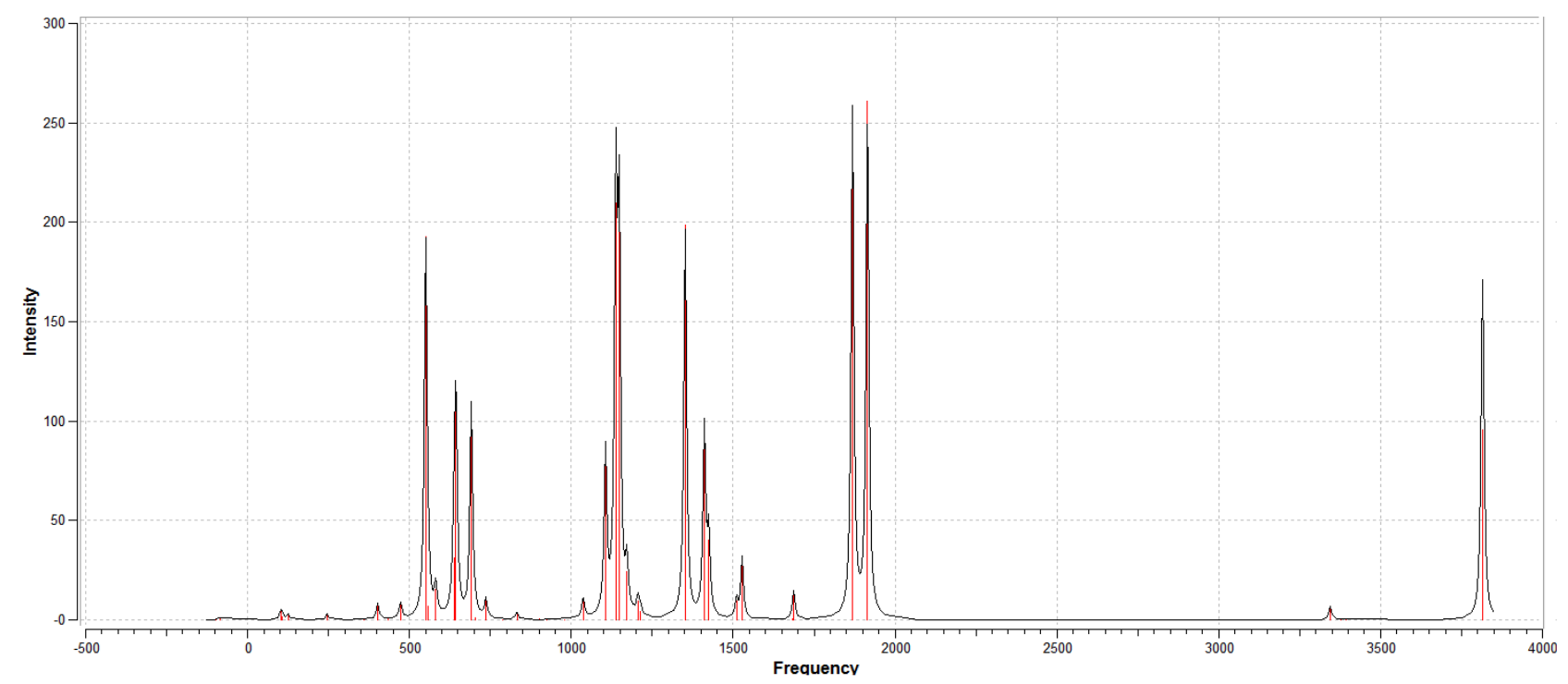

(a)

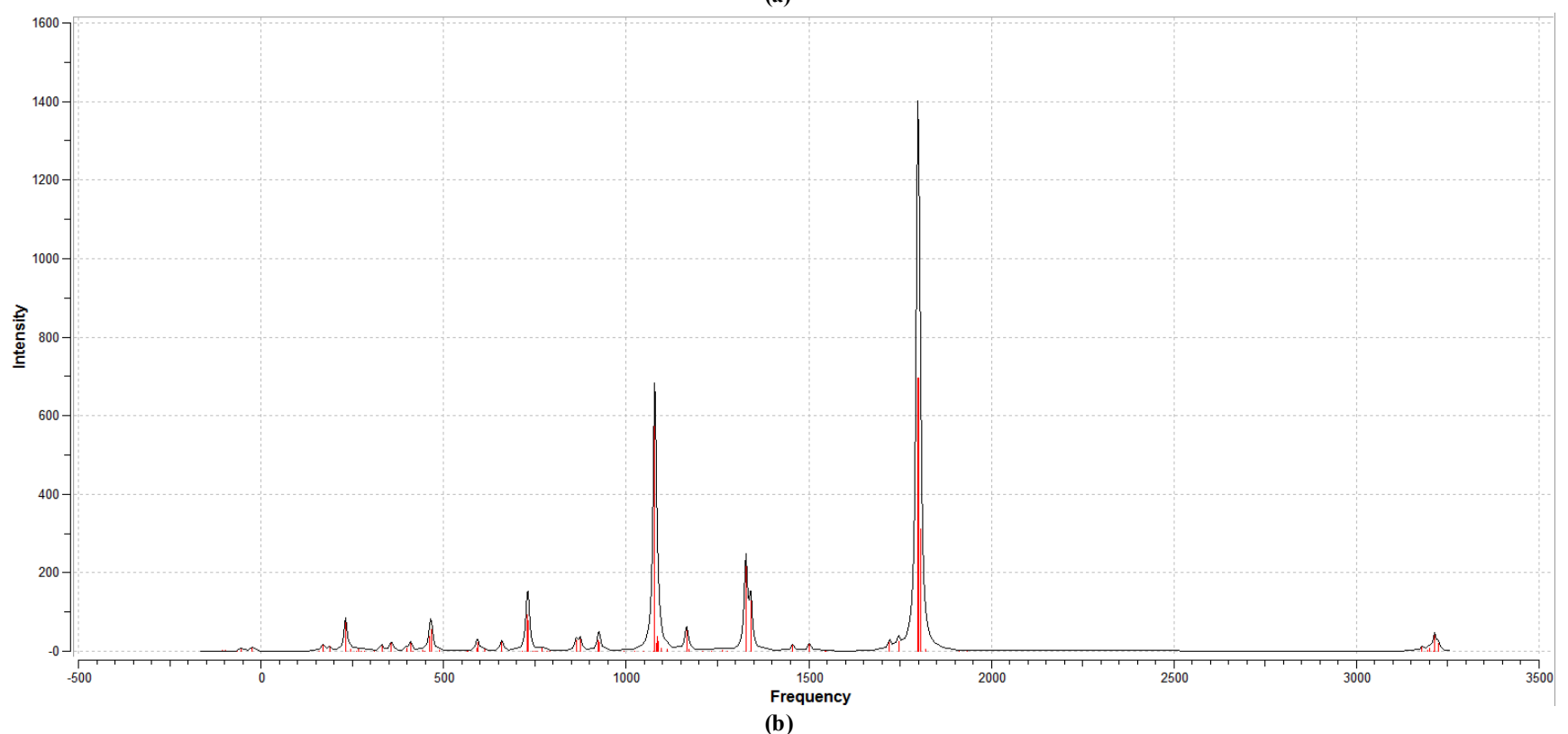

Figure 4. FTIR spectrum (a) the free ligand and (b) Fe-PDC

In order to shed more light on the reaction Fenton-like mechanis $m$ and to understand the role of Fe-PDC in this catalytic process, theoretical calculations were performed. Initially, a potential energy surface along the dihedral $\mathrm{C}-\mathrm{N}-\mathrm{Fe}-\mathrm{N}$ was carried out to identify the transition structures and intermediaries involved. In an $a b$ initio calculation of the type reported here, there is a primary consideration: the choice of the basis set and the form of the exchange-correlation functional. From our calculation, a good agreement between the calculated and experimental geometry for complex Fe-PDC was observed with the theoretical approach used.

Our theoretical results indicate that the chemical bond between oxygen and the iron atom in the Fe-PDC complex is predominantly electrostatic, small variation along the dihedral C-N-Fe-N are favored with little energy cost. Thus, a chemical bond between iron and hydroxyl peroxide is feasibly. In this line, the hydrogen peroxide coordinates with iron atom. We can, in principle, justify the formation of hydro xyl radicals, because direct coordination of Fe with peroxide molecule lead to formation of a reactive peroxo-comp lex (Figure 5).

The generated peroxo-complex (Figure 6) has two possible reaction pathways. The first one, is the formation of the $\cdot \mathrm{OOH}+\mathrm{H}^{+}$species and the second one is the formation of $\bullet \mathrm{OH}+\mathrm{OH}^{-}$. From our calculations, it is clear that second way is more stable thermodynamically.

It is well-known that the Kohn-Sham DFT is a leading method for electronic structure calculations in chemistry mainly due to its high efficiency and relatively low computational cost $[59,60]$. It is important to mention that the orbital concept at the DFT level is questionable theoretically[16, 20-22]. In fact, some authors have analysed the behavior of $\mathrm{HF}$ and $\mathrm{KS}$ orbital energies in 
many molecules and compared the results with the experimental IP, showing that KS orbital energies differ significantly from experimental IP, while HF energies are in good agreement with them[56]. On the other hand, our previous findings[23] pointed out that KS orbitals are suitable for being used in qualitative MO theory in combination with the FERMO concept. It should also be kept in mind, however, that, despite the recent improvements in DFT, there are still difficulties in using DFT to properly describe intermolecular interactions, especially van der Waals forces (dispersion) and charge transfer excitations, in part, due to lack of exact Hartree-Fock exchange in some functionals [62-65].

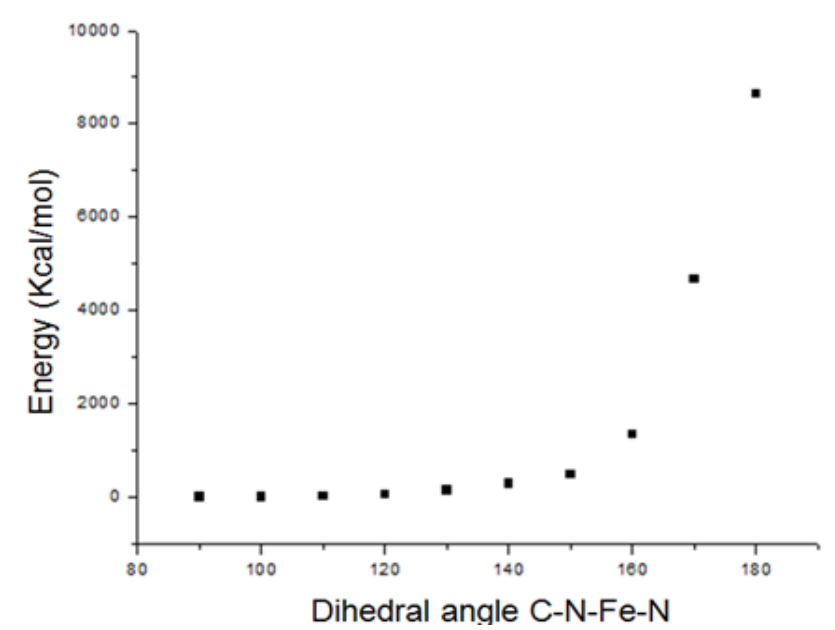

Figure 5. PES for the Fe-PDC complex structure
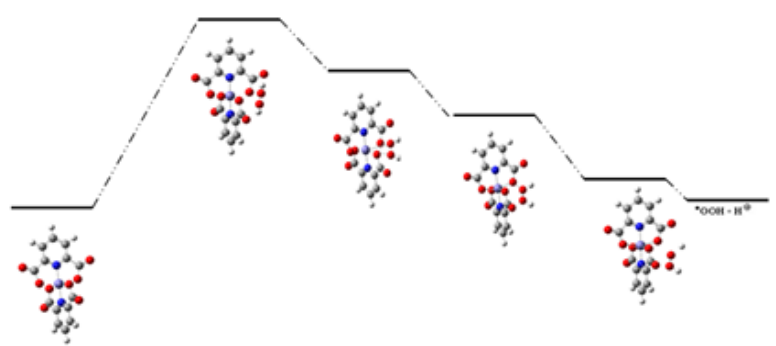

(a)
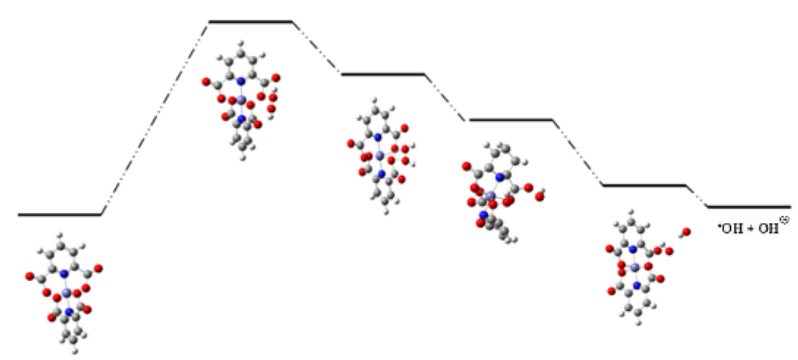

(b)

Figure 6. Catalytic cycle proposed model structures

Currently, the development of methods designed to overcome this problem, by alterations to the functional or by the inclusion of additive terms, is a very important research topic in DFT. It is also well-known that the hybrid functionals, such as the B3LYP functional used in this work, yield unsatis factory atomization energies ma in ly for metals, whose the error can vary between $25 \%$ and $40 \%$ [62-64].

On the other hand, for large gap systems with well-localized electrons, the agreement with experimental and conventional functionals is quite reasonable[65,66]. In this way, our results using B3LYP combined with the FERMO concept could then be justified, because the respective errors for the constituents of the reaction cancel each other, as electrons are localized for all reactants and the product[23-30]. This conclusion is in line with previous work of other groups [67,68].

Thus, we may notice that the FERMO idea is to use the MO calculations with a valence bond theory (VB) interpretation. In MO theory, the electrons in a molecule occupy delocalized orbitals made from linear combination of AOs. However, it should be kept in mind that the VB approaches are quite useful in analyzing enzymatic reactivity[69,70] photochemistry[70,71], chemical dynamics,[71] and theories of conductivity, where the localized representation seemed indispensable.

In fact, it is well known that the VB methods allow generating new ideas on chemical bonding. Recently, there has been an intense surge of concepts related to localized representation, for example, multiple bonding between transition metals $[13,72,73]$ and the development of natural resonance theory[74].

In this previous study[30], the molecular dynamics direct calculations rein forces the idea of the FERMO concept, and leads us to new perspectives on the role of frontier orb itals. Because the molecular orbital responsible for the chemical bond formation has electrons with higher kinetic energy.

Another interesting factor that we observe is that the shape of molecular orbital effective for the reaction (FERMO) is maintained throughout the reaction. So, the FERMO concept might remove the limitations of the HOMO-LUMO argument, employing a small dose of chemical intuition.

For instance, the Pearson's Hard and Soft Acid-Base Principlecan also invoke the FERMO concept. The hardness of a molecule is defined as the difference between HOMO and LUMO energy, thus, according to that definition, only a single value of hardness would exist for a given molecule. By using the FERMO concept, we can identify different values each reaction site.

\section{Conclusions}

Overall, the FERMO concept has been successfully applied to describe to the Pearson's hard and soft principle in a very simple and chemically intuitive way. The results of this study demonstrated that the complex of iron and acid dipicolínico solid is a good catalyst for the oxidation of quinoline by a Fenton-like mechanis $\mathrm{m}$. The results obtained by first principles calculation suggest that proposed mechanis $\mathrm{m}$ is thermodynamic favorable. Vibrational 
analysis confirm the formation of the complex Fe-PDC compared with the free ligand spectrum. Thus, the FERMO concept cay be useful to explain acid-base reactions in a wide range of applications. Nevertheless, it is the FERMO concept (the shape of the reactant orbital) that really provided chemical insight about which is the $\mathrm{MO}$ related to acid-base reactions. Understanding the behavior of the molecular orbital is crucial to understand the chemistry.

\section{ACKNOWLEDGEMENTS}

We thank the Brazilian agencies CAPES, CNPq and FAPEMIG for funding part of this work. We also are especially grateful to the CEANAPD-SP for the computational facilities.

\section{REFERENCES}

[1] Eric Largy, Florian Hamon, FrédéricRosu, ValérieGabelica, Edwin De Pauw, AuroreGuédin Jean-Louis Mergny, Marie-PauleTeulade-Fichou, "Tridentate N-Donor Palladium(II) Complexes as Efficient Coordinating Quadrup lex DNA Binders", Chemistry - A European Journal, vol.17, no.47, pp.13274-13283, 2011.

[2] Tomona Yutaka, Shinya Obara, Satoshi Ogawa, Koichi Nozaki, Noriaki Ikeda, Takeshi Ohno, Youichi Ishii, Ken Sakai, Masa-akiHaga, "Syntheses and Properties of Emissive Iridium(III) Complexes with Tridentate Benzimidazole Derivatives“, Inorganic Chemistry , vol.44, no.13, pp.4737-4746, 2005.

[3] Hudson W P Carvalho, Ana P L Batista, Peter Hammer, Gustavo H P Luz, Teodorico C Ramalho, "Removal of Metal Ions from Aqueous Solution by Chelating Polymeric Hydrogel", Environmental Chemistry Letters, vol. 8, no.4, pp. 343-348, 2010 .

[4] Plinio Di Bernardo, Andrea Melchior, MarilenaTolazzi, Pier Luigi Zanonato, "Thermodynamics of Lanthanide(III) Complexation in non-Aqueous Solvents", Coordination Chemistry Reviews, vol.256, no.1-2, pp.328-351, 2012.

[5] ElifGungor, Selma Celen, DilekAzaz, Hulya Kara, "Two Tridentate Schiff Base Ligands and their Mononuclear Cobalt (III) Complexes: Synthesis, Characterization, Antibacterial and Antifungal Activities", SpectrochimicaActa Part A, vol.94, pp.216-221, 2012.

[6] Julio R Sambrano, Marcelo Zampieri, Antonio G Ferreira, Elson Longo, "Ab initio study and NMR analysis of the complexion of citric acid with ion lithium", Journal of Molecular Strucuture (THEOCHEM), vol.493, pp.309-318, 1999.

[7] Colin R Andrew, Joann Sanders-Loehr, "Copper-Sulfur Proteins: Using Raman Spectroscopy to Predict Coordination Geometry", Accounts of Chemical Research, vol.29, no.8, pp.365-372, 1996.

[8] J Mitchell Guss, Hans C Freeman, "Structure of Oxidized Poplar Plastocyanin at $1.6 \AA$ resolution", Journal of Molecular Biology, vol.169, no.2, pp.521-563, 1983.

[9] Cristiano Benelli, Dante Gatteschi, "Magnetism of
Lanthanides in Molecular Materials with Transition-Metal Ions and Organic radicals", Chemical Reviews, vol. 6, no. 102, pp.2369-2387, 2002.

[10] Mark P Lowe, David Parker, "pH Switched Sensitisation of $\mathrm{Eu}(\mathrm{III})$ by a Dansyl Group" InorganicaChimicaActa, vol.317,no.1-2, pp.163-173, 2001.

[11] Ken Kuriki, Yasuhiro Koike, Yoshi Okamoto, "Plastic Optical Fiber Lasers and Amplifiers Containing Lanthanide Complexes", Chemical Reviews, vol.102, no.6, pp.2347-2356, 2002.

[12] Jagannath B Lamture, Theodore G Wensel, "A Novel Reagent for Labelling Macromolecules with Intensely Luminescent Lanthanide Complexes", Tetrahedron Letters, vol. 34,no.26, pp. 4141-4144, 1993.

[13] Kenichi Fukui, Teijiro Yonezawa , HaruoShingu, "A Molecular Orbital Theory of Reactivity in Aromatic Hydrocarbons", Journal of Chemical Physics, vol.20, no.4, pp.722-725, 1952.

[14] Kenichi Fukui , TeijiroYonezawa , Chikayoshi Nagata," Molecular Orbital of Orientation in Aromatic, Heteroaromatic and other Conjugates Molecules", Journal of Chemical Physics, vol.22, no.8, pp. 1433-1442, 1954.

[15] Kenichi Fukui, "The Role of Frontier Orbitals in Chemical Reactions", AngewandteChemie International Edition in English, vol.21, no.11, pp. 801-809, 1982.

[16] Roald Hoffmann, Robert B Woodward, "Conservation of Orbital Symmetry", Accounts of Chemical Research", vol.1, no.1, pp. 17-22, 1968.

[17] Robert Vianello, Zvonimir B Maksić, "Triadic Analysis of Substituent Effects-Gas-Phase Acidity of para-Substituted Phenols", Tetrahedron, vol.62, no.14, pp. 3402-3411, 2006.

[18] Hajime Hirao, TomohikoOhwada , "Theoretical Study of Reactivities in Electrophilic Aromatic Substitution Reactions: Reactive Hybrid Orbital Analysis", Journal of Physical Chemistry A, vol.107, no.16, pp. 2875-2881, 2003.

[19] Hajime Hirao, TomohikoOhwada, "Theoretical Revisit of Regioselectivities of Diels-Alder Reactions: Orbital-Based Reevaluation of Multicentered Reactivity in Terms of Reactive Hybrid Orbitals", Journal of Phy sical Chemistry A, vol.109, no.5, pp. 816-824, 2005.

[20] Hiroshi Fujimoto, Yoshitaka Mizutani, Koji Iwase, “An Aspect of Substituents and Peripheral Structures in Chemical Reactivities of Molecules", Journal of Physical Chemistry, vol. 90, no.12, pp.2768-2772, 1986.

[21] Hiroshi Fujimoto, "Paired interacting orbitals: a way of looking at chemical interactions", Accounts of Chemical Research, vol.20, no.12, pp. 448-453, 1987.

[22] Hiroshi Fujimoto, Shinichi Satoh "Orbital Interactions and Chemical Hardness“, Journal of Phy sical Chemistry, vol. 98, no.5, pp.1436-1441, 1994.

[23] Rodrigo R da Silva, Teodorico C Ramalho, Joana M Santos, J Daniel Figueroa-Villar, "On the Limits of Highest-Occupied Molecular Orbital Driven Reactions: The Frontier Effective-for-Reaction Molecular Orbital Concept", Journal of Physical Chemistry A, vol.110, no.3, pp. 1031-1040, 2006.

[24] Rodrigo R Da Silva, Joana M Santos, Teodorico C Ramalho, J Daniel Figueroa-Villar," Concerning The FERMO Concept 
and Pearson's Hard and Soft Acid-Base Principle", Journal of the Brazilian Chemical Society, vol.17, no.2, pp.223-226,2006.

[25] Rodrigo R da Silva, Teodorico C Ramalho, Joana M Santos, J Daniel Figueroa-Villar, "Comment on the Paper 'On the Limits of Highest-Occupied Molecular Orbital Driven Reactions: The Frontier Effective-for-Reaction Molecular Orbital Concept", Journal of Physical Chemistry A, vol. 110, no.36, pp.10653-10654, 2006.

[26] Douglas H Perreira, Teodorico C Ramalho , "Understanding the Substituent Effect on the Acidity of Alcohols and Para-Substituted Phenols", Molecular Simulation, vol. 35, no.15, pp.1269-1278, 2009.

[27] Edson Barbosa da Costa, Milan Trsic, "A Quantum Chemical Study on a Set of Non-Imidazole H3 Antihistamine Molecules", Journal of Molecular Graphics and Modelling, vol. 28, no.7, pp. 657-663, 2010.

[28] Felipe A La Porta, Regis T Santiago, Teodorico C Ramalho, Matheus P Freitas, Elaine F F Da Cunha, "The Role of The Frontier Orbitals in Acid-Base Chemistry of Organic Amines Probed by ab Initio and Chemometric Techniques", International Journal of Quantum Chemistry, vol. 110, no.15, pp. 2015-2023, 2010.

[29] Regis T Santiago, Felipe A La Porta, Marcus V J Rocha, Teodorico C Ramalho, Matheus P Freitas, Elaine F F da Cunha, "Description of the Acid/Base Behavior of Organic Phosphines Using ab initio and Chemometric Approaches", Letters in Organic Chemistry, vol.7, no.7, pp.552-556, 2010.

[30] Felipe A La Porta, Teodorico C Ramalho, Régi s T Santiago, Marcus V J Rocha, Elaine F F da Cunha," Orbital Signatures as a Descriptor of Regioselectivity and Chemical Reactivity: The Role of the Frontier Orbitals on 1,3-Dipolar Cycloadditions", Journal of Physical Chemistry A, vol.115, no.5, pp.824-833, 2011.

[31] Pierre Hohenberg, Walter Kohn, "Inhomogeneous Electron Gas", Physical Review, vol.136, no. 3B, pp.B864-B871, 1964.

[32] Walter Kohn, Lu Jeu Sham, "Self-Consistent Equations Including Exchange and Correlation Effects", Physical Review, vol.140, no.4A, pp.A1133-A1138, 1965.

[33] M J Frisch, G W Trucks, H B Schlegel, G E Scuseria, M A Robb, J R Cheeseman, V G Zakrzewski, J A Montgomery Jr., R E Stratmann, J C Burant, S Dapprich, J MMillam, A D Daniels, K. N. Kudin, M. C. Strain, O. Farkas, J. Tomasi, VBarone, M Cossi, R Cammi, B Mennuchi, CPomelli, CAdamo, S Clifford, JOchterski, G A Petersson, P Y Ayala, Q Cui, K Morokuma, DK Malick, A D Rabuck, K Raghavachari, J B Foresman, J Cioslowski, J V Ortiz, A G Baboul, B BStefanov, G Liu, A Liashenko, P Piskorz, I Komaromi, R Gomperts, R LMartin, D J Fox, T Keith, M A Al-Laham, C Y Peng, A Nanayakkara, C Gonzalez, M Challacombe, P M W Gill, B G Johnson, W Chen, M W Wong, J L Andres, M Head-Gordon, E SReplogle, J APople, Gaussian 98 (Revision A.9), Gaussian, Inc., Pittsburgh, PA (1998).

[34] Axel D Becke, "Density-Functional Thermochemistry. III. The role of exact exchange", Journal of Chemical Physics, vol. 98 , no.7, pp.5648-5652, 1993

[35] Chengteh Lee, Weitao Yang, Robert G. Parr, "Development of the Colle-Salvetti correlation-Energy Formula into a Functional of the Electron Density", Physical Review B,
vol.B37, no.2, pp.785-789, 1988.

[36] Charles W Bauschlicher, "Large Atomic Natural Orbital Basis Sets for the First Transition Row Atoms, TheoreticaChimicaActa, vol. 92, no.3, pp.183-198, 1995.

[37] Lydia Rhymana, Hassan H Abdallahb, Sabina Jhaumeer-Laullooa, Luis R Domingoc, John A Jouled, PonnaduraiRamasami, "1,3-Dipolar Cycloaddition of 1H-Pyrazinium-3-olate and N1- and C-methyl substituted pyrazinium-3-olates with Methyl Acrylate: A Density Functional Theory Study", Tetrahedron, vol.67, no.43, pp.8383-8391, 2011.

[38] Teodorico C Ramalho, Douglas H Pereira, Walter Thiel," Thermal and Solvent Effects on NMR Indirect Spin-Spin Coupling Constants of a Prototypical ChagasDisease Drug", Journal of Physical Chemistry A, vol.115, no.46, pp.13504-13512, 2011.

[39] Melissa S Caetano, Teodorico C Ramalho, Douglas F Botrel, Elaine F F da Cunha, Walclee C. Mello, "Understanding the Inactivation Process of Organophosphorus Herbicides: A DFT Study of Glyphosate Metallic Complexes with $\mathrm{Zn}^{2+}$, $\mathrm{Ca}^{2+}, \mathrm{Mg}^{2+}, \mathrm{Cu}^{2+}, \mathrm{Co}^{3+}, \mathrm{Fe}^{3+}, \mathrm{Cr}^{3+}$, and $\mathrm{Al}^{3+}$, International Journal of Quantum Chemistry, vol.112, no.15, pp.2752-2762, 2012.

[40] Martina E Judmaier, Andreas Wallner, Gregor N Stipicic, Karl Kirchner, Judith Baumgartner, Ferdinand Belaj, Nadia C Mösch-Zanetti, "Molybdenum(VI) dioxo Complexes With Tridentate Phenolate Ligands", Inorganic Chemistry, vol.48, no.21, pp. 10211-10221, 2009.

[41] Patrícia P Lima, Oscar L Malta, Severino A Junior, "Estudo Espectroscópico de Complexos de Eu (III), Tb (III) e Gd (III) com Ligantes Derivados de Ácidos Dicarboxílicos", Química Nova, vol. 28, no.5, pp.805-808, 2005.

[42] Xiang-Li Wang, Hui Chao, Hong Li, Xian-LanHonga, Liang-NianJi, Xiao-Yuan Li, "Synthesis, Crystal Structure and DNA CLEAVAGE ACTIVITIES of Copper(II) Complexes with Asymmetric Tridentate Ligands", Journal of Inorganic Bioch emistry vol.98, no.3, pp.423-429, 2004.

[43] VitalieStavila, Kenton H Whitmire, Irene Rusakova, "Synthesis of Bi2S3 Nanostructures from Bismuth (III) Thiourea and Thiosemicarbazide Complexes", Chemistry of Materials, vol.21, no.22, pp.5456-5465, 2009.

[44] J F Hsu, J J Chen, C C Chuang, H H Wei, M C Cheng, Y Wang, Y DYau, "Crystal Structure, Magnetic Property and Mossbauer Spectra of bis(Pyridine-2,6-Dicarboxylato) Iron(III) Dihy drate", Inorgan icaChimicaActa, vol. 184, no.1, pp. 1-5, 1991.

[45] MadhumitaChatterjee, Milan Maji, SaktiprosadGhosh, Thomas C W Mak, "Studies of V(III) Complexes with Selected A-N-Heterocy clic Carboxy lato NO Donor Ligands: Structure of a New Seven-Coordinated Pentagonal Bipyramidal Complex Containing Picolinato Ligands", Journal of the Chemical Society, no.21, pp.3641-3646, 1998.

[46] Ana C González- Baró, Eduardo E Castellano, Oscar E Piro, Beatriz S Parajón Costa, "Synthesis, Crystal Structure and Spectroscopic Characterization of a Novel bis (oxo-bridged) dinuclear vanadium(V)-dipicolinic acid complex”, Poly hedron, vol.24, no.1,pp.49-55, 2005.

[47] Philippe Lainé, André Gourdon, Jean-Pierre Launay, "Chemistry of Iron with Dipicolinic Acid. 1. Mononuclear 
Complexes of Iron(II) or Iron(III)", Inorganic Chemistry, vol.34, no.21, pp. 5129-5137, 1995.

[48] Ralph G Pearson," Hard and Soft Acids and Bases", Journal of the American Chemical Society, vol.85, no.22, pp.3533-3539, 1963.

[49] Gilles Klopman, "Chemical Reactivity and the Concept of Charge- and Frontier-Controlled Reactions", Journal of the American Chemical Society, vol.90, no.2, pp.223-234, 1968.

[50] Sadaf Khan, Shahab A ANami , K S Siddiqi, Eram Husain, ImranaNaseem, "Synthesis and Characterization of Transition Metal 2,6-pyridinedicarboxylic Acid Derivatives, Interactions of $\mathrm{Cu}(\mathrm{II})$ and $\mathrm{Ni}(\mathrm{II})$ Complexes with DNA in vitro “, SpectrochimicaActa A, vol.72, no.2, pp.421-428, 2009.

[51] Debbie C Crans, Luqin Yang, TamasJakusch, Tamas Kiss, "Aqueous Chemistry of Ammonium (Dipicolinato)oxovanadate(V): The First Organic Vanadium(V) Insulin-Mimetic Compound Inorganic Chemistry, vol. 39, no.20, pp. 4409-4416, 2000.

[52] Richard J Watts, Samuel E Dilly, "Evaluation of Iron Catalysts for the Fenton-Like Remediation of Diesel-Contaminated Soils", Journal of Hazardous Materials, vol.51, no.1-3, pp.209-224, 1996.

[53] Raquel F P Nogueira, Alam G Trovó, Milady Renata A da Silva, Ricardo D Villa, Mirela C de Oliveira, "Fundamentos e Aplicações Ambientais dos Processos Fenton e foto-Fenton”, Química Nova, vol.30, no.2, pp.400-,2007.

[54] Pier G. Daniele, Concetta De Stefano, Enrico Prenesti, Silvio Sammartano, "Copper(II) Complexes of N-(phosphonomethyl)Glycine in Aqueous Solution: A Thermodynamic and Spectrophotometric Study", Talanta, vol. 45, no.2, pp.425-431, 1997.

[55] Beatriz S Parajón-Costa, Oscar E Piro, ReinaldoPis-Diez, Eduardo E Castellano, Ana C. González-Baró, "Crystal Structures, Spectroscopic Characterization and Theoretical Calculations of the Guanidinium and Ammonium Salts of the Insulin-Enhancing Anion [VO2(dipic)]"“, Polyhedron, vol.25, no.15, pp.2920-2928, 2006.

[56] Teodorico C Ramalho, Tales L C Martins, Luiz Eduardo, Pizarro Borges, José D Figueroa-Villar, "Influence of Nonbonded Interactions in the Kinetics Of Formation of Chalcogenol Esters from Chalcogenoacetylenes", International Journal of Quantum Chemistry, vol.95, no.3, pp.267-273, 2003.

[57] Xue Wang, K N Houk, Martin Spichty, Thomas Wirth, "Origin of Stereoselectivities in Asymmetric Alkoxy seleneny lations ", Journal of the American Chemical Society, vol.121, no.37, pp.8567-8576, 1999.

[58] Richard E Rosenfeld Jr., RParthasarathy, Jack D Dunitz,'Directional preferences of nonbonded atomic contacts with divalent sulfur. 1. Electrophiles and nucleophiles", Journal of the American Chemical Society, vol.99, no.14, pp.4860-4862, 1977.

[59] David R. Lide, CRC Handbook of Chemistry and Physics: A Ready-Reference Book of Chemical and Physical Data, 88th ed., CRC Press: Boca Raton FL, 2008.

[60] Andrea Vittadini, Maurizio Casarin, AnnabellaSelloni, "Chemistry of and on $\mathrm{TiO}_{2}$ Anatase Surfaces by DFT
Calculations: a Partial Review", Theoretical Chemistry Accounts, vol.117, no.5-6, pp.663-671, 2007.

[61] Peter Politzer, Fakher Abu-Awwad, "A Comparative Analy sis of Hartree-Fock and Kohn-Sham Orbital Energies", Theoretical Chemistry Accounts, vol.99, no.2, pp.83-87, 1998.

[62] Joachim Paier, MartijnMarsman, Kerstin Hummer, Georg Kresse, I C Gerber, Janos G Ångyán, "Screened Hybrid Density Functionals Applied to Solids", Journal of Chemical Physics, vol.124, no.15, pp.154709-154721, 2006.

[63] Teodorico C Ramalho, Carlton A Taft, "Thermal and Solvent Effects on the NMR and UV Parameters of Some Bioreductive Drugs", Journal of Chemical Physics, vol.123, no.5, pp. 54319-54325, 2005.

[64] Joachim Paier, MartijnMarsman, Georg Kresse, "Why does the B3LYP Hybrid Functional Fail for Metals?", Journal of Chemical Physics, vol.127, no2, pp. 024103-124112, 2007.

[65] Frank De Proft, Paul Geerlings, "Conceptual and Computational DFT in the Study of Aromaticity" Chemical Reviews, vol.101, no.5, pp.1451-1464, 2001.

[66] Teodorico C Ramalhoa, Luiz C A Oliveira, Kele T G Carvalho, Eu gênio F Souza, Elaine F F da Cunha, Marcelo Nazzaro, "The Molecular Basis for the Behaviour of Niobia Species in Oxidation Reaction Probed by Theoretical Calculations and Experimental Techniques", Molecular Physics, vol.107, no.2, pp.171-179, 2009.

[67] GianlucaCiancaleoni, NatasciaFraldi, Peter H M Budzelaar, Vincenzo Busico, Roberta Cipullo, AlceoMacchioni, "Structure-Activity Relationship in Olefin Polymerization Catalysis: Is Entropy the Key?", Journal American Chemical Society, vol.132, no.39, pp.13651-13653, 2010.

[68] Sérgio F Sousa, Pedro A Fernandes, Maria J Ramos, "General Performance of Density Functionals", Journal of Physical Chemistry, vol. 111, no.42, pp.10439-10453, 2007.

[69] Hisao Nakamura, Donald G. Truhlar," The Direct Calculation of Diabatic States Based on Configurational Uniformity", Journal of Chemical Physical, vol.115, no.22, pp.10353-10372.

[70] Donald G Truhlar, "Valence Bond Theory For Chemical Dynamics", Journal of Computational Chemistry, vol.28, no.1, pp 73-86, 2007.

[71] MarcinBrynda, Laura Gagliardi, Per-OlofWidmark, Philip P. Power, Björn O. Roos, "A Quantum Chemical Study of the Quintuple Bond between Two Chromium Centers in [PhCrCrPh]: trans-Bent versus Linear Geometry", AngewandteChemie International Edition, vol.45, no.23, pp.3804-1807, 2006.

[72] Frank Weinhold, Clark R. Landis, "High Bond Orders in Metal-Metal Bonding”, Science, vol.316, no.5821, pp.61-63, 2007.

[73] Karl Jug, Philippe C Hiberty, SasonShaik, " $\sigma-\pi$ Energy Separation in Modern Electronic Theory for Ground States of Conjugated Systems", Chemical Reviews, vol.101, no.5, pp.1477-1500, 2001.

[74] Vladimir Georgescu, Christian Gerard, "On the Virial Theorem in Quantum Mechanics“, Communications in Mathematical Physics, vol.208, no.2, pp.275-281, 1998. 